\title{
Teaching Colonoscopy: Scope of Practice or Practice of Scope?
}

\author{
Joseph C. Yarze $e^{1,2}$ \\ Accepted: 27 October 2021 / Published online: 19 November 2021 \\ (C) The Author(s), under exclusive licence to Springer Science+Business Media, LLC, part of Springer Nature 2021
}

\section{Background}

Teaching colonoscopy can be tedious, yet much more rewarding than many appreciate. I allude to teaching "colonoscopy" and not only "colonoscopic technique" for a reason. The last 30 years have taught me that there is an order of magnitude greater knowledge needed to master the art of colonoscopy in contradistinction to simply passing the colonoscope tip to the cecum. My career began at a large multispecialty clinic where, at the time, I had little to offer as I was an attending "supervisor" as opposed to an endoscopic "trainer" [1]. Over the subsequent 25 years, I learned the nuances of colonoscopic technique. I realized the benefit of the insertion tube hanging loosely from the stretcher edge and the necessity of working with a straightened colonoscope, as well as the technique of how to ensure the same upon scope insertion.

\section{Definitions and Scheduling Considerations}

Over the past 4 years, I have been privileged to be an endoscopic educator at a large tertiary referral center. Understanding that no single approach is universally successful or superior, below are techniques I presently employ in order to assist my serving as a mentor or trainer as opposed to a supervisor [2]. In addition to defining an endoscopic mentor or trainer as one who explicitly enhances the skills of the learner, I more simply define this task as someone whom the fellow perceives to be a catalyst to reaching new levels. I am the primary endoscopic trainer for 5 senior fellows each year, spending one half day in the endoscopy unit with each of these fellows performing outpatient procedures that are

Joseph C. Yarze

JYARZE@mgh.harvard.edu

1 GI Unit, Massachusetts General Hospital, Director of Fellowship Endoscopic Training, Boston, MA, USA

2 Harvard Medical School, Boston, MA, USA scheduled every 35 min (bidirectional examinations every $70 \mathrm{~min}$ ). These procedures are performed employing moderate sedation (or occasionally no sedation). Although it has never been studied, I suspect that fellows who learn colonoscopy employing moderate sedation may become more facile by necessity. I limit the number of procedures scheduled in a session (6 in a half day); time at the end of a session is kept open as a buffer. I attempt to not unduly rush the fellow with whom I am working, and I make every attempt to not keep another endoscopist waiting to begin their session.

\section{Prior to the Procedure/Setting the Stage for Success}

During the initial session of my engagement with a fellow, I allow them to observe me performing several procedures. During this session, we review endoscopy unit etiquette and the importance of appropriate positive interaction with the endoscopy team (nurse and technician) and patients. While exuding a polite demeanor, it is made clear that an endoscopy session is not the appropriate forum for an intensive social engagement. Distractions are kept to an absolute minimum; in particular, I refrain from performing unrelated tasks while the fellow is performing a procedure. Though I emphasize that the overwhelming majority of GI endoscopic procedures proceed uneventfully, having an evervigilant mindset to the possibility of an imminent unanticipated event will serve the endoscopist and their patient well. When the chips are down (so to speak), it is essential to have a team engaged in a mindful and inter-reliant manner. If I note a team member having difficulty completing a task, I always attempt to lend assistance without being asked. If one adopts this approach, reciprocation is more likely. I emphasize that checking the equipment prior to each procedure is the fellow's responsibility. After all, even though mechanics keep an airplane in excellent working order, it is always the pilot's ultimate responsibility to check the aircraft prior to each flight. The fellow understands that it is their responsibility to review the patient's medical record and understand 
the indication for which the procedure is being performed. The fellow also obtains informed consent for the procedure, although prior to a procedure commencing, I always personally ensure that there are no outstanding questions or concerns. Occasionally I will observe the fellow while they obtain informed consent, providing feedback with a focus on viewing the consent process through the patient's lens, especially as it pertains to the use of inappropriate medical jargon.

\section{Detailed Approach to Colonoscopic Technique}

\section{Pre-procedure}

The importance of employing favorable ergonomics is stressed prior to starting the procedure [3]. The video monitor is positioned so that the endoscopist is looking downward at a $\sim 15^{\circ}$ angle in order to avoid neck strain. I ensure that the patient is positioned optimally, with the buttocks close to the edge of the stretcher. The advantages to this are twofold: First, the insertion tube can then hang freely, and when hand torque is applied, one is not sensing resistance, as occurs when the scope lays on the stretcher. Second, in this position, the endoscopist is standing upright and not leaning forward. I insist upon application of the water immersion technique, the benefits of which have previously been described in detail [4].

\section{Practical Considerations}

Early on, I emphasize the importance of keeping the insertion tube straight (i.e., unlooped) as the primary method of ensuring patient comfort. In this manner, the use of escalating doses of sedation and analgesia is less often required. My preference is to use an adult colonoscope for all cases, unless I have had personal experience that dictates otherwise. I am usually not hampered by utilizing an adult scope even in small patients (of either sex) with a history of previous pelvic surgery. An adult colonoscope is also less prone to looping - the main culprit in causing traction on the mesentery, and subsequent pain.

\section{The Procedure Itself}

\section{General Considerations}

Although other methods have been described, I only espouse the torque steering technique as the primary method of colonoscope insertion [5]. Fellows are encouraged (and constantly reminded) to keep the right hand on the insertion tube. I relate that the use of gentle right-hand torque and maximal use of the up-down deflector should be the usual approach employed. With practice, most angulations can be negotiated in this fashion. As the technique becomes further refined, the use of the left-right deflector (to accentuate tip deflection after maximal use of the up-down deflector) becomes less necessary and is viewed as a rescue maneuver. With the scope hanging off the stretcher, if I must momentarily take my right hand off the insertion tube, I then use my right thigh to stabilize scope position. On rare occasion, when necessary, I will drop down to either a pediatric or ultra-thin instrument in order to traverse a very tight angulation or stricture.

\section{Keeping the Scope Straight}

Early on, it is emphasized that there is usually a superior manner to approach a turn that is less likely to be associated with distal loop formation. This is sensed by considering the luminal view, gauging the tension on the insertion tube, and most importantly, what is occurring with the external portion of the insertion tube. When turns are traversed favorably, there is less right-hand torque required; the colonoscope continues to hang loosely with little sense of tension. If while inserting the colonoscope an external loop is noted, the insertion tube is promptly rotated in the proper direction so as to straighten and reduce this loop using clockwise or counterclockwise torque. If something unfavorable is occurring with the insertion tube externally, it is likely compensating for something unfavorable internally. A frequent to and fro movement of the well-lubricated colonoscope assists in keeping the scope straight, since the colon is pleated onto the insertion tube. I withdraw a bit in order to ensure a straightened scope position at least several times during the insertion phase in keeping with the observation that "the most proficient colonoscopist withdraws the scope more than they insert it." If one becomes facile with the aforementioned aspects of colonoscope insertion, stiffening the insertion tube, application of abdominal counterpressure and patient repositioning are used much less frequently. When these adjunctive measures are required, they should be rapidly employed in a stepwise fashion since repeating the same unsuccessful maneuver is frowned upon. Though paradoxical movement is considered proof of a loop, the converse is not necessarily true. Early on in their training, though many fellows suggest that the scope is in the straightened position surmised from a positive 1 to 1 movement, the colonoscope tip is actually in the transverse colon and $100 \mathrm{~cm}$ of the scope is within the patient. In this situation, I am certain that a distal loop is present. My simple rule of thumb is: If the colonoscope tip is in the cecum, there is good 1 to 1 movement and $70 \mathrm{~cm}$ or so of the insertion tube is within the patient, the scope is straight, with rare exceptions. 


\section{lleal Intubation}

I insist upon ileal intubation in the overwhelming majority of situations (including screening examinations). Though the technique of ileal intubation is considered a "procedure within a procedure," if proper technique is solidified and practiced frequently, the endoscopist will have a greatly enhanced success rate when ileal intubation is deemed crucial. As with any endoscopic maneuver, the importance of a straight scope position is stressed at the outset. The cecum is then deflated a bit (to keep things short), the valve is positioned (using torque) at the 6 or 12 o'clock position, and the scope is then withdrawn as the up-down deflector is gradually and simultaneously engaged to allow the scope to "drop into the ileum." Driving the scope forward into the ileum from above is discouraged as in most instances, this approach is associated with a distal loop beginning to form. The orientation of the ileocecal valve (ICV) opening can usually be accurately predicted by the shape of the appendiceal orifice, and from there, having the scope proximal to the ICV orifice and trusting the up-down deflector as the scope is withdrawn a bit is all that is usually required. Parenthetically, for both ileal intubation and facilitating retroflexion in the ascending colon, if initially unsuccessful, rotating the insertion tube by 180 degrees will almost universally be met with success during both maneuvers [6].

\section{Enhancing the Adenoma Detection Rate}

In order to enhance the adenoma detection rate, I expect use of at least one and usually a combination of two of the following: examination of the ascending colon twice on anterograde view, ascending colon retroflexion, and/or employment of a colonoscope cap attachment [7]. I want fellows to be acquainted with these adjunctive approaches so that ultimately they can choose the one or more with which they are most comfortable.

\section{Rectal Retroflexion}

The importance of developing sound technique to perform comfortable rectal retroflexion is emphasized since after all, this is the last thing a patient might remember. I advocate withdrawing the scope to the anal verge and then generously lubricating the insertion tube. In most circumstances, the scope is then advanced toward the most distal rectal valve while simultaneously employing greater tip deflection with only the up-down deflector (toward me). I attempt to avoid torquing the scope until it is in the fully retroflexed position. The left-right deflector (toward me) is then employed to accentuate the tip deflection so as to gain a closer view of the anal verge. Only at this point is the scope torqued to obtain a panoramic view on both sides of the insertion tube. All too often, excessive torque is applied before the scope is fully retroflexed, a maneuver associated with a less than comfortable situation. Given the above, performing retroflexion in the ascending colon and rectum is different. In the ascending colon, I employ simultaneous advancement of the insertion tube and application of counterclockwise torque to complete the retroflexion. In the rectum, torque is only applied to the insertion tube after the scope is retroflexed. When any therapeutic maneuver is performed, the importance of straight scope position and having the region of concern at the 5 o'clock position (where the opening of the operating channel is situated) is stressed.

\section{Teaching Approach}

Fellows are allowed to be challenged, but not struggle. It is understood that maintaining patient safety and comfort are always the overarching goal. Interestingly, fellows with whom I work are accustomed to ask for assistance quite early. They have learned from former trainees that when difficulty is experienced, there is usually an approach that is more comfortable, efficient, and elegant. In general, I initially demonstrate a maneuver while lending a verbal description of the deconstructed task. When the same issue presents itself again, I then offer less hands-on (and more verbal) assistance until before long, the trainee can complete the maneuver independently, while they provide me with a verbal description of how they are completing the task [8]. I have uniformly noted that fellows who are eager observers are those who have the steepest learning curve and ultimately become the most proficient graduate endoscopists. Thus, passing the scope to the mentor for demonstration of a maneuver should be framed as a vital learning opportunity much rather than as a sign of failure, since the ultimate goal for the fellow is not to merely complete a procedure but to do so in the safest, most efficient, and comfortable manner. After each session (and usually after each procedure) there is a brief review of what has occurred, stressing the positive, but also suggesting where there is an opportunity for improvement. I also probe to inquire the trainee's perspective in these regards. This bidirectional reflection and assessment are keys. A text in the evening is not infrequently sent after I have also had the opportunity to review my own performance if there were any particularly challenging cases where my intervention was necessary. A formal, written summative evaluation is provided quarterly so that fellows can gauge their success and always have a clear path for improvement. 


\section{Conclusion}

In the final analysis, the above are some techniques that I presently employ when performing and teaching colonoscopy. Over the last three decades, my approach has admittedly evolved; the importance of continuous, critical self-evaluation, and a strong desire to be a student of the colonoscopic procedure cannot be overstressed. If nothing else, I hope that I have instilled the importance of appropriate perseverance and the need to continually refine one's approach to the fellows I am privileged to train.

\section{References}

1. Yarze JC. Transitioning from the mountains to the Mecca: one gastroenterologist's journey. Dig Dis Sci. 2021 (in press).

2. Waschke KA, Anderson J, Valori RM et al. ASGE principles of endoscopic training. Gastrointest Endosc. 2019;90:27-34.
3. Singla M, Kwok RM, Deriban G et al. Training the endo-athlete: an update in ergonomics in endoscopy. Clin Gastroenterol Hepatol. 2018;16:1003-1006.

4. Leung CW, Kaltenbach T, Soetikno R et al. Water immersion versus standard colonoscopy insertion technique: randomized trial shows promise for minimal sedation. Endoscopy 2010;42:557-563.

5. Williams CB. Insertion techniques. In: Waye JD, Rex DK, Williams CB, eds. 2nd edn. Chichester, UK: Blackwell Publishing Ltd; 2009; P537-P559.

6. Yarze JC. An adjunctive maneuver to facilitate colonoscopic retroflexion in the right colon. Am J Gastroenterol. 2019;115:304.

7. Shaukat A, Kahi CJ, Burke CA et al. ACG clinical guidelines: colorectal cancer screening 2021. Am J Gastroenterol. 2021;116:458-479.

8. Peyton JWR. Teaching and learning in medical practice, Herts: Manticore Europe; 1998: p 216.

Publisher's Note Springer Nature remains neutral with regard to jurisdictional claims in published maps and institutional affiliations. 